\title{
Inflammatory factors are associated with early neurological deterioration in acute ischemic stroke patients receiving endovascular therapy-The AISRNA study
}

\section{Qi-Wen Deng}

Nanjing First Hospital https://orcid.org/0000-0003-4324-8009

Shi Huang

Nanjing First Hospital

Shuo Li

Southeast University

Qian Zhai

Nanjing First Hospital

Qing Zhang

Nanjing First Hospital

Zhen-Jie Wang

Nanjing First Hospital

Wen-Xia Chen

Nanjing First Hospital

Hong-Chao Shi

Nanjing First Hospital

\section{Feng Zhou}

Nanjing First Hospital

Hui-Ling Sun ( $\nabla$ sunhuiling1988@yeah.net)

Nanjing First Hospital

Min Lu ( $\square$ luminnfhsjk@126.com )

Nanjing First Hospital

Jun-Shan Zhou ( $\square$ zhjsh333@126.com )

Nanjing First Hospital

\section{Research}

Keywords: acute ischemic stroke; early neurological deterioration; endovascular therapy; inflammatory factors; IL-6; IL-4 
Posted Date: May 11th, 2020

DOl: https://doi.org/10.21203/rs.3.rs-27300/v1

License: (c) (i) This work is licensed under a Creative Commons Attribution 4.0 International License. Read Full License 


\section{Abstract}

Background: This study aimed to explore several peripheral blood-based markers related to inflammatory response in a total of 85 patients with acute ischemic stroke (AIS) caused by large artery occlusion in the anterior circulation receiving endovascular therapy (EVT) from the AISRNA study regarding the association between inflammatory factors and early neurological deterioration (END), and investigated whether their time course correlated with END after EVT.

Methods: We collected baseline characteristics of 85 AIS patients participating in an observational acute stroke cohort: the AISRNA study. The following inflammatory factors were measured in these participants: interleukin-2 [IL-2], IL-4, IL-6, IL-10, tumor necrosis factor-a [TNF-a], and interferon- $\mathrm{\text {[IFN- }} \gamma$ ]. The National Institute of Health Stroke Scale score increase of $\geq 4$ within 24 hours after EVT defined as END.

Results: IL-6 and IL-10 were higher in patients with END compared to those with non-END $(P<0.01)$, and they were also associated with risk factors of END after EVT. Furthermore, we found that the area under curves (AUCs) of IL-6 and IL-10 for predicting END were 0.791 (0.689-0.871), and 0.564 (0.452-0.671), respectively. Adjusting for age, sex, and atrial fibrillation, the odds ratios (ORs; $95 \%$ confidence interval) for incident END for IL-6 and IL-10 were 1.83 (1.08-6.36) and 1.15 (1.02-1.30), respectively. Additionally, we found significant changes over time in the expression levels of IL-4, IL-6, and IL-10 in patients undergoing END compared with non-END $(P<0.05)$.

Conclusions: IL- 6 and IL-10 levels on admission are significantly associated with END after EVT, and time course of IL-4, IL-6, and IL-10 is correlated with stroke progression. Further study of molecular mechanisms on peripheral immunomodulation in AIS would be helpful.

Trial registration: ClinicalTrials.gov NCT04175691. Registered November 21, 2019.

\section{Introduction}

Stroke is commonly considered as a major cause for adult disability and mortality. Although the early advent of endovascular therapy (EVT) has obviously improved clinical outcome of acute ischemic stroke (AIS), over 50 percent of patients still suffer from disabilities and deficits, which may be the result of neurological and medical complications (1). Precise prediction of clinical outcome remains challenging during the acute phase of ischemic stroke (IS) after EVT. While our previous studies have found that demographic and several clinical characteristics are associated with prognosis after acute ischemic stroke (2-4), the accuracy of prediction remains limited, especially during the acute phase of IS (5). Therefore, development of precise scores to predict prognosis in the acute stage may benefit from the individual biomarkers.

The correlation between stroke and acute inflammatory response can be found in various stages of acute infection. Accumulating evidence has indicated that stroke induces a rapid immunodepression through 
the autonomic nervous system $(6,7)$. Immune factors including cytokines are indicative to the strokeassociated infection and associated with clinical outcome after AIS $(8,9)$. However, the link between inflammatory factors and stroke progression remains unclear in patients receiving EVT.

In the present study, we aimed to explore the association between a range of inflammation-related biomarkers (interleukin-2 [IL-2], IL-4, IL-6, IL-10, tumor necrosis factor-a [TNF-a], and interferon-y [IFN- $]$ ]) and early neurological deterioration (END) after stroke caused by large artery occlusion in the anterior circulation. We enrolled of a total of 85 patients receiving EVT from the AISRNA study to investigate the link between inflammatory factors and END, and observed that high levels of IL- 6 and IL-10 were associated with END. Additionally, time course of IL-4, IL-6, and IL-10 is correlated with stroke progression after EVT.

\section{Materials And Methods}

\section{Study population}

Our study protocol was approved by the Nanjing Medical University Ethics Committee and followed the Declaration of Helsinki. We enrolled 85 AIS patients caused by large artery occlusion in the anterior circulation receiving EVT in the present study. Firstly, a total of 85 AIS patients receiving EVT were prospectively collected from the AISRNA study (www.clinicaltrials.gov, NCT04175691) to investigate the association of inflammatory factors on admission and their dynamic changes with END for 7 days. Their clinical characteristics are summarized in Table 1. All patients were from Nanjing First Hospital, Nanjing Medical University which is a stroke center affiliated to China Stroke Association. Eligible patients were collected in the present study if they followed these inclusion criteria: (1) AIS patients receiving EVT; (2) the anterior circulation occlusion; (3) followed standard in-house procedures (4); and exclusion criteria: (1) preexisting dysphagia; (2) intracranial hemorrhage; (3) infection on admission; (4) antibiotic or immunodepression medical therapy within 4 weeks; (5) age less than 18 years; (6) history of a malignant tumor; (7) died within 7 days.

\section{Clinical data}

Demographics, medical history, stroke severity on admission and 24 hours, stroke etiology, laboratory parameters, modified Thrombolysis in Cerebral Infarction Score [mTICl], and time of onset to door, groin puncture, and reperfusion. Several data definitions were used in the present study: the National Institute of Health Stroke Scale (NIHSS) was used to assess stroke severity; the delta NIHSS scale (delta NIHSS 0$24 \mathrm{~h}$ ) = NIHSS on admission - NIHSS after 24 hours; early neurological deterioration (END) was defined as $\triangle$ NIHSS $\geq 4$ points (10); stroke etiology was determined according to the Trial of Org 10172 in acute stroke treatment (TOAST) criteria (11).

\section{Inflammation-related biomarkers}


Blood samples were collected within 24 hours after admission (day 1) and repeated on days 2, 3 and 7. Plasma samples were centrifuged $\left(2000 \mathrm{rpm}, 4^{\circ} \mathrm{C}, 20 \mathrm{~min}\right)$ and stored at $-80^{\circ} \mathrm{C}$. Plasma concentrations of IL-2, IL-4, IL-6, IL-10, TNF-a, and IFN- $y$ were determined with the Navios flow cytometer (Beckman Coulter, California, USA). The upper limit of detection is $2500 \mathrm{pg} / \mathrm{mL}$ for these factors.

\section{Statistical analysis}

Categorical variables are expressed as frequency and percentage. Continuous variables of an abnormal distribution are expressed as medians (interquartile range [IQR]), and normally distributed variables as mean \pm standard deviation (SD). The $t$-test, one-way ANOVA and Mann-Whitney U-tests were used to analyze continuous variables if necessary. Bivariate correlation between delta NIHSS score and inflammatory factors was analyzed by the Spearman correlation. Variables from significant factors of the univariable logistic regression analysis were considered in the multivariate logistic regression model to obtain independent factors. Discriminatory capacities of inflammatory factors was assessed by receiver operating characteristic (ROC) curve analyses and area under the curve (AUC). The significance threshold was set at less than 0.05 .

\section{Results}

Of 553 patients screened from the AISRNA study between November 2019 and April 2020, a total of 85 participants (34 female; mean age, $66.95 \pm 12.5$ years) met the inclusion criteria (Fig. 1). Four hundred and sixty-eight patients were excluded according to the following criteria: lack of EVT $(n=437)$, the posterior circulation $(n=9)$, died within 7 days $(n=3)$, preexisting dysphagia $(n=2)$, intracranial hemorrhage $(n=3)$, infection on admission $(n=4)$, antibiotic or immunodepression therapy within 4 weeks $(n=3)$, and no blood samples $(n=5)$. Baseline characteristics of the eligible patients are summarized in Table 1. Of the 85 patients included, 37 (43.5\%) received intravenous thrombolysis.

A total of $10(11.8 \%)$ patients underwent END after EVT. The association between inflammatory factors on admission and incidence of END is shown in Table 1. The NIHSS on admission was no significant difference in both groups $(P=0.378)$. However, compared with non-END, NIHSS was significantly higher in patients with END after 24 hours $(P<0.001)$. We found that END was associated with increased IL-6 and IL-10 levels as well as higher proportion of atrial fibrillation $(P<0.05)$. As the delta NIHSS was considered as END, we performed a correlation analysis to investigate the correlation between inflammatory factors and delta NIHSS $0-24 \mathrm{~h}$. The results showed that the delta NIHSS was correlated with the expression of IL-6 $(r=0.260, P=0.016)$ and IL-10 ( $r=-0.238, P=0.028)$ (Fig. 2B and 2C). Additionally, we performed a ROC curve analysis to explore their predictive powers. We observed that the AUCs of IL- 6 and IL-10 for predicting END were 0.791 (0.689-0.871), and 0.564 (0.452-0.671), respectively (Fig. 3), revealing that IL-6 outperformed IL-10 in predicting END $(P<0.001)$.

Given that association of inflammatory factors with END, we conducted a binary logistic regression to analyze the impact of inflammatory factors on END. The results of the univariate analyses showed that 
IL-6 and IL-10 levels were prognostic factors for END, and the findings remained stable after adjustment (Table 2).

To further study the clinical utility of inflammatory factors after EVT, we analyzed time courses for inflammatory factors within 7 days for END and non-END (Fig. 4). Among these patients, there were 85 samples for day 1 (on admission), 58 for day 2 , and 28 for day 3 and 7 . We found significant changes over time in the expression levels of IL-4, IL-6, and IL-10 in patients undergoing END compared with nonEND $(P<0.05)$, and IL-6 levels were obviously increased from days 1 to 7 after EVT (Fig. 4B, $P<0.001$ ). Furthermore, we observed IL-4 and IL-10 peak levels at 2 days, and then rapidly decreased at 3 days. Additionally, patients with END had decreased IL-10 levels compared with those with non-END at 7 days (Fig. 4C, $\mathrm{P}<0.01$ ).

\section{Discussion}

This study of EVT participants from the AISRNA study showed a strong association of IL-6 and IL-10 levels on admission with risk of END. Our study suggested that patients with increased IL-6 and IL-10 levels had higher risks of developing post-EVT END. By contrast, there was no correlation of baseline levels of IL-2, II-4, INF- $\gamma$, and TNF-a with END. The predictive power of IL-6 for END was superior to that of other inflammatory factors. Additionally, IL-4, IL-6, and IL-10 levels presented a marked rising trend in patients with END.

Inflammation is a hallmark of stroke etiology and progression. Post-stroke inflammation is accounted as a requisite pathological process involved in ischemic brain injury (12). A series of detrimental complications occur and the blood-brain barrier (BBB), damaged after the initial brain injury, crossed by activated peripheral immune cells including monocytes and T-cells (13). Furthermore, post-stroke inflammatory response is associated with stroke severity on admission as determined by NIHSS (14). Additionally, patients who underwent END were more susceptible to have a poor functional outcome after 90 days (15). However, the association between post-stroke inflammatory response and END remains uncertain. Therefore, we performed a prospective study from the AISRNA study to further investigate the influence of inflammatory factors on END after stroke.

The biological function of IL-6 in AIS remains unclear. Mounting evidence have demonstrated that IL- 6 levels are increased in peripheral blood samples during the first 7 days after stroke onset. For example, increased IL-6 was associated with the infarct size and stroke severity on admission (16) as well as risk of incident stroke (17), although a study reported the opposite that early IL-6 levels, as a neuroprotective factor, was inversely correlated with lesion size and functional outcome (18). Our findings also suggested that increased IL- 6 was associated with risk of END after EVT. However, there is a less agreement on the time point of peak IL- 6 levels. Some studies reported IL-6 peak levels at 3 day $(19,20)$, which are similar to our findings. Other describe high levels of IL-6 with ranged from a few hours till one day or first week after stroke $(18,21,22)$. 
We also reported positive correlation between IL-10 and END after EVT. Previous studies have suggested IL-10 as a marker for incident stroke $(23,24)$. IL-10 is a major anti-inflammatory cytokine to regulate the immune response by suppressing pro-inflammatory cytokine expression. The influence of IL-10 on functional outcome and its time course after EVT remains uncertain. Several investigators reported that IL-10 may serve a neuroprotective role and predict clinical outcome after stroke $(25,26)$. However, our previous studies from the experimental rats and human stroke patients observed that IL-10 was positively associated with stroke risk $(27,28)$. In the current study, increased IL-10 levels on admission were associated with END after EVT. Whereas we observed IL-10 peak levels at 2 days, and patients with END had decreased IL-10 levels compared with those with non-END at 7 days. This phenomenon may be due to a stress response of IL-10 regarding END which lead high levels of IL-10, but its levels were subsequently decreased after 2 days. Therefore, IL-10 may be involved in the process of END, and its molecular mechanisms remain to be explored in the further study.

IL-4, an anti-inflammatory cytokine, can drive Th2 cell differentiation which plays beneficial roles in inhibiting post-stroke inflammation, repairing damaged brain tissues and inducing neurotrophic factors in astrocytes $(29,30)$. A previous study has demonstrated that IL-4 was significantly correlated with stroke severity and functional outcome in AIS patients (31). However, our results showed no association of IL-4 with END after EVT, but IL-4 levels were increased in patients with END at 2 days and then rapidly decreased at 3 days. At present, we are also investigating its molecular mechanisms regarding dynamic change of IL-10 in stroke progression.

Several limitations should be acknowledged in the study. First, this is a small number of patients in a single center, but we are seeking for sub-centers to complete the AISRNA study. Second, we did not analyze the association between initial infarct burden and END after EVT. Finally, molecular mechanisms regarding dynamic changes of these inflammatory factors should be further explored in patients undergoing EVT.

\section{Conclusions}

In summary, this study illustrates the influence of inflammatory factors on END and their time course after EVT among AIS patients caused by large artery occlusion in the anterior circulation. We found that serum concentration of IL-6 and IL-10 on admission is obviously associated with END after EVT, and time course of these factors is correlated with stroke progression. Thus, peripheral immunomodulation in AIS remains to be further studied to provide a new pharmacological approach with stroke therapy.

\section{Abbreviations}

END, early neurological deterioration; IL, interleukin; TNF, tumor necrosis factor; INF, interferon; BMI, body mass index; DM, diabetes mellitus; CAD, coronary artery disease; AF, atrial fibrillation; IS, ischemic stroke; 
TIA, transient ischemic attack; IQR, interquartile range; $\mathrm{mTICl}$, modified Thrombolysis in Cerebral Infarction Score; NIHSS, National Institute of Health Stroke Scale; LAA, large artery atherosclerosis; TC, total cholesterol; TG, triglyceride; LDL-C, low-density lipoprotein cholesterol; HDL-C, high-density lipoprotein cholesterol; hs-CRP, high-sensitivity C-reactive protein.

\section{Declarations}

\section{Ethics approval and consent to participate}

This study protocol was approved by the ethics committee of Nanjing First Hospital, Nanjing Medical University and followed the tenets of the Declaration of Helsinki. Written informed consent was obtained from all individuals or their family members. This study design is based on the stroke registry of Nanjing First Hospital, Nanjing Medical University, a national advanced stroke center affiliated with Stroke Prevention Project, National Health Commission.

\section{Consent for publication}

Not applicable.

\section{Availability of data and materials}

All data supporting our results are available from the corresponding authors upon reasonable.

\section{Declaration of interests}

The authors declare that they have no competing interests.

\section{Author's contributions}

Data curation, Qi-Wen Deng, Shi Huang, and Shuo Li; Formal analysis, Hong-Chao Shi and Feng Zhou; Investigation, Qian Zhai, Qing Zhang, Zhen-Jie Wang, and Wen-Xia Chen; Project administration, Hui-Ling Sun and Min Lu; Writing - original draft, Qi-Wen Deng and Min Lu; Writing - review \& editing, Hui-Ling Sun, Min Lu and Jun-Shan Zhou.

\section{Acknowledgements}

None.

\section{Funding}

This work was supported by the National Natural Science Foundation of China [No. 81901215 (to Qi-Wen Deng) and No. 81802093 (to Hui-Ling Sun)], the Nanjing Medical University Science and Technique Development Foundation Project [No. NMUB2018325 (to Qi-Wen Deng)], the Science and Technology Development Plan of Nanjing Foundation [No. 201727004 (to Hong-Chao Shi) and the Nanjing Medical Science and Technique Development Foundation Project [No. YKK18097 (to Feng Zhou)]. 


\section{References}

1. Hankey GJ. Stroke. Lancet. 2017;389(10069):641-54.

2. Deng QW, Liu YK, Zhang YQ, Chen XL, Jiang T, Hou JK, et al. Low triglyceride to high-density lipoprotein cholesterol ratio predicts hemorrhagic transformation in large atherosclerotic infarction of acute ischemic stroke. Aging. 2019;11(5):1589-601.

3. Deng QW, Li S, Wang H, Lei L, Zhang HQ, Gu ZT, et al. The Short-term Prognostic Value of the Triglyceride-to-high-density Lipoprotein Cholesterol Ratio in Acute Ischemic Stroke. Aging disease. 2018;9(3):498-506.

4. Deng QW, Wang H, Sun CZ, Xing FL, Zhang HQ, Zuo L, et al. Triglyceride to high-density lipoprotein cholesterol ratio predicts worse outcomes after acute ischaemic stroke. Eur J Neurol. 2017;24(2):283-91.

5. Byblow WD, Stinear CM. It Is Difficult to Make Predictions. Especially About the Future Stroke. 2017;48(12):3187-8.

6. Chamorro A, Urra X, Planas AM. Infection after acute ischemic stroke: a manifestation of braininduced immunodepression. Stroke. 2007;38(3):1097-103.

7. Deng QW, Yang H, Yan FL, Wang H, Xing FL, Zuo L, et al. Blocking Sympathetic Nervous System Reverses Partially Stroke-Induced Immunosuppression but does not Aggravate Functional Outcome After Experimental Stroke in Rats. Neurochem Res. 2016;41(8):1877-86.

8. Schuppner R, Dirks M, Grosse GM, Bockmann M, Goetz F, Pasedag T, et al. ADAMTS-13 Activity Predicts Outcome in Acute Ischaemic Stroke Patients Undergoing Endovascular Treatment. Thromb Haemost. 2018;118(4):758-67.

9. Bustamante A, Sobrino T, Giralt D, Garcia-Berrocoso T, Llombart V, Ugarriza I, et al. Prognostic value of blood interleukin- 6 in the prediction of functional outcome after stroke: a systematic review and meta-analysis. J Neuroimmunol. 2014;274(1-2):215-24.

10. Seners P, Hurford R, Tisserand M, Turc G, Legrand L, Naggara O, et al. Is Unexplained Early Neurological Deterioration After Intravenous Thrombolysis Associated With Thrombus Extension? Stroke. 2017;48(2):348-52.

11. Adams HP Jr, Bendixen BH, Kappelle LJ, Biller J, Love BB, Gordon DL, et al. Classification of subtype of acute ischemic stroke. Definitions for use in a multicenter clinical trial. TOAST. Trial of Org 10172 in Acute Stroke Treatment. Stroke. 1993;24(1):35-41.

12. Chamorro A, Hallenbeck J. The harms and benefits of inflammatory and immune responses in vascular disease. Stroke. 2006;37(2):291-3.

13. Esenwa CC, Elkind MS. Inflammatory risk factors, biomarkers and associated therapy in ischaemic stroke. Nature reviews Neurology. 2016;12(10):594-604.

14. Chamorro A, Amaro S, Vargas M, Obach V, Cervera A, Gomez-Choco M, et al. Catecholamines, infection, and death in acute ischemic stroke. J Neurol Sci. 2007;252(1):29-35. 
15. Kim JM, Bae JH, Park KY, Lee WJ, Byun JS, Ahn SW, et al. Incidence and mechanism of early neurological deterioration after endovascular thrombectomy. J Neurol. 2019;266(3):609-15.

16. Hotter B, Hoffmann S, Ulm L, Meisel C, Fiebach JB, Meisel A. IL-6 Plasma Levels Correlate With Cerebral Perfusion Deficits and Infarct Sizes in Stroke Patients Without Associated Infections. Front Neurol. 2019;10:83.

17. Jenny NS, Callas PW, Judd SE, McClure LA, Kissela B, Zakai NA, et al. Inflammatory cytokines and ischemic stroke risk: The REGARDS cohort. Neurology. 2019;92(20):e2375-e84.

18. Sotgiu S, Zanda B, Marchetti B, Fois ML, Arru G, Pes GM, et al. Inflammatory biomarkers in blood of patients with acute brain ischemia. Eur J Neurol. 2006;13(5):505-13.

19. Perini F, Morra M, Alecci M, Galloni E, Marchi M, Toso V. Temporal profile of serum anti-inflammatory and pro-inflammatory interleukins in acute ischemic stroke patients. Neurological sciences: official journal of the Italian Neurological Society of the Italian Society of Clinical Neurophysiology. 2001;22(4):289-96.

20. Hoffmann S, Harms H, Ulm L, Nabavi DG, Mackert BM, Schmehl I, et al. Stroke-induced immunodepression and dysphagia independently predict stroke-associated pneumonia - The PREDICT study. J Cereb Blood Flow Metab. 2017;37(12):3671-82.

21. Mazzotta G, Sarchielli P, Caso V, Paciaroni M, Floridi A, Floridi A, et al. Different cytokine levels in thrombolysis patients as predictors for clinical outcome. Eur J Neurol. 2004;11(6):377-81.

22. Smith CJ, Emsley HC, Gavin CM, Georgiou RF, Vail A, Barberan EM, et al. Peak plasma interleukin-6 and other peripheral markers of inflammation in the first week of ischaemic stroke correlate with brain infarct volume, stroke severity and long-term outcome. BMC Neurol. 2004;4:2.

23. van Exel E, Gussekloo J, de Craen AJ, Bootsma-van der Wiel A, Frolich M, Westendorp RG. Inflammation and stroke: the Leiden 85-Plus Study. Stroke. 2002;33(4):1135-8.

24. Xie G, Myint PK, Zaman MJ, Li Y, Zhao L, Shi P, et al. Relationship of serum interleukin-10 and its genetic variations with ischemic stroke in a Chinese general population. PLoS One. 2013;8(9):e74126.

25. Protti GG, Gagliardi RJ, Forte WC, Sprovieri SR. Interleukin-10 may protect against progressing injury during the acute phase of ischemic stroke. Arq Neuropsiquiatr. 2013;71(11):846-51.

26. Singh HV, Pandey A, Shrivastava AK, Raizada A, Singh SK, Singh N. Prognostic value of neuron specific enolase and IL-10 in ischemic stroke and its correlation with degree of neurological deficit. Clin Chim Acta. 2013;419:136-8.

27. Li S, Lu G, Wang D, He JL, Zuo L, Wang H, et al. MicroRNA-4443 regulates monocyte activation by targeting TRAF4 in stroke-induced immunosuppression. Eur J Neurol. 2020.

28. Wang H, Deng QW, Peng AN, Xing FL, Zuo L, Li S, et al. beta-arrestin2 functions as a key regulator in the sympathetic-triggered immunodepression after stroke. J Neuroinflammation. 2018;15(1):102.

29. Xiong X, Barreto GE, Xu L, Ouyang YB, Xie X, Giffard RG. Increased brain injury and worsened neurological outcome in interleukin-4 knockout mice after transient focal cerebral ischemia. Stroke. 2011;42(7):2026-32. 
30. Pena-Philippides JC, Caballero-Garrido E, Lordkipanidze T, Roitbak T. In vivo inhibition of miR-155 significantly alters post-stroke inflammatory response. J Neuroinflammation. 2016;13(1):287.

31. Li X, Lin S, Chen X, Huang W, Li Q, Zhang H, et al. The Prognostic Value of Serum Cytokines in Patients with Acute Ischemic Stroke. Aging disease. 2019;10(3):544-56.

\section{Tables}

Table 1. Baseline characteristics of the eligible patients stratified by END

\begin{tabular}{|c|c|c|c|c|}
\hline & total & Non-END & END & $\mathrm{p}$-value \\
\hline & $\mathrm{n}=85$ & $\mathrm{n}=75$ & $\mathrm{n}=10$ & \\
\hline \multirow[t]{3}{*}{ ) } & $34(40.0)$ & 29 (38.7) & $5(50.0)$ & 0.492 \\
\hline & $66.95 \pm 12.5$ & $66.52 \pm 13.0$ & $70.20 \pm 8.0$ & 0.387 \\
\hline & $24.96 \pm 3.8$ & $24.71 \pm 3.76$ & $26.81 \pm 3.5$ & 0.098 \\
\hline \multicolumn{5}{|l|}{$I$} \\
\hline \multirow[t]{5}{*}{ on } & $57(67.1)$ & $48(64.0)$ & $9(90.0)$ & 0.199 \\
\hline & $17(20.0)$ & $15(20.0)$ & $2(20.0)$ & 0.674 \\
\hline & $12(14.1)$ & $11(14.7)$ & $1(10.0)$ & 0.932 \\
\hline & $20(23.5)$ & $15(20.0)$ & $5(50.0)$ & 0.036 \\
\hline & 14 (16.5) & $10(13.3)$ & $4(40.0)$ & 0.093 \\
\hline \multicolumn{5}{|l|}{$\overline{\mathrm{a}}$} \\
\hline & $41(48.2)$ & $36(48.0)$ & $5(50.0)$ & 0.315 \\
\hline \multirow[t]{2}{*}{$\mathrm{sm}$} & $31(36.5)$ & $26(34.7)$ & $5(50.0)$ & \\
\hline & $13(15.3)$ & $13(17.3)$ & $0(0.0)$ & \\
\hline ission & $12(6-17.5)$ & $14(12-19)$ & 18 (7.75-28.5) & 0.378 \\
\hline \multirow[t]{2}{*}{ th } & $15(12-20)$ & $11(5-15)$ & $31(19.5-36)$ & $<0.001$ \\
\hline & $37(43.5)$ & $32(42.7)$ & $5(50.0)$ & 0.660 \\
\hline \multicolumn{5}{|l|}{ nin, median (IQR) } \\
\hline in puncture & $103.5(81.5-130)$ & $101.5(85-130)$ & 109 (62.5-178.5) & 0.697 \\
\hline \multirow[t]{2}{*}{ erfusion } & $229.49 \pm 194.6$ & $180(149-219)$ & $239(118.8-302.5)$ & 0.585 \\
\hline & $67(78.8)$ & $60(80.0)$ & $7(70.0)$ & 0.753 \\
\hline \multicolumn{5}{|l|}{ ever } \\
\hline & $56(65.9)$ & $52(69.3)$ & $4(40.0)$ & 0.138 \\
\hline & $29(34.1)$ & $23(30.7)$ & $6(60.0)$ & \\
\hline \multicolumn{5}{|c|}{ actors on admission } \\
\hline 3$)$ & $2.47 \pm 1.1$ & $2.45 \pm 1.1$ & $2.61 \pm 1.3$ & 0.653 \\
\hline -) & $3.29 \pm 1.4$ & $2.83 \pm 1.8$ & $3.05 \pm 1.5$ & 0.718 \\
\hline ) & $12.13(7.18-25.82)$ & $10.00(6.67-22.52)$ & $26.94(17.88-117.69)$ & 0.005 \\
\hline $\mathrm{iL})$ & 4.65 (3.07-6.62) & 4.65 (3.15-6.65) & $5.02(2.69-23.3)$ & 0.001 \\
\hline 'mL) & $2.16(1.44-3.05)$ & $2.13(1.44-2.92)$ & $2.29(1.23-4.35)$ & 0.360 \\
\hline $\mathrm{mL})$ & $2.18(1.53-3.08)$ & $2.15(1.57-3.09)$ & $2.24(0.88-3.18)$ & 0.554 \\
\hline \multicolumn{5}{|l|}{ aracteristics } \\
\hline \multirow{2}{*}{$\mathrm{g} / \mathrm{mL})$} & $5.05(4.46-11.62)$ & $5.05(4.44-11.80)$ & 5.21 (4.24-12.09) & 0.579 \\
\hline & $5.80(5.48-6.53)$ & $5.75(5.40-5.60)$ & $6.05(5.60-6.43)$ & 0.667 \\
\hline ucose $(\mathrm{mmol} / \mathrm{L})$ & $6.46(5.10-8.08)$ & $6.41(5.09-8.09)$ & $7.03(6.14-8.19)$ & 0.521 \\
\hline \multirow[t]{2}{*}{$\mathrm{mol} / \mathrm{L})$} & $67.0(58.0-88.6)$ & $67.0(58.0-80.1)$ & $64.5(55.8-96.0)$ & 0.599 \\
\hline & $1.18(0.83-1.81)$ & $1.28(0.87-1.84)$ & $0.83(0.67-1.52)$ & 0.368 \\
\hline (L) & $4.30 \pm 1.3$ & $4.37 \pm 1.4$ & $3.76 \pm 0.7$ & 0.168 \\
\hline $1 / \mathrm{L})$ & $0.96(0.82-1.16)$ & $0.91(0.80-1.16)$ & $1.05(0.89-1.24)$ & 0.534 \\
\hline$/ / \mathrm{L})$ & $2.64 \pm 1.0$ & $2.69 \pm 1.0$ & $2.23 \pm 0.7$ & 0.175 \\
\hline
\end{tabular}

END, early neurological deterioration; IL, interleukin; TNF, tumor necrosis factor; INF, interferon; BMI, body mass index; DM, diabetes mellitus; $\mathrm{CAD}$, coronary artery disease; $\mathrm{AF}$, atrial fibrillation; IS, ischemic stroke; TIA, transient ischemic attack; IQR, interquartile range; mTICI, modified Thrombolysis in Cerebral Infarction Score; NIHSS, National Institute of Health Stroke Scale; LAA, large artery atherosclerosis; TC, total cholesterol; TG, triglyceride; LDL-C, low-density lipoprotein cholesterol; HDL-C, high-density lipoprotein cholesterol; hs-CRP, high-sensitivity Creactive protein; IVT, intravenous thrombolysis.

$\Delta$ NIHSS 0-24h was defined as the delta NIHSS scale (delta NIHSS 0-24h) = NIHSS on admission - NIHSS after 24 hours. 
Continuous variables of abnormal distribution are expressed as medians (interquartile range), and normally distributed variables as mean \pm standard deviation (SD). Categorical variables are expressed as frequency and percentage.

a According to the modified TOAST classification.

Table 2. Logistic regression analyses for inflammatory factors associated with END

\begin{tabular}{|l|c|c|c|}
\hline Variable & Model $^{\text {a }}$ & OR (95\% confidence interval) & p-value \\
\hline & 1 & $1.14(0.64-2.04)$ & 0.649 \\
\hline & 2 & $\ldots$ & $\ldots$ \\
\hline & 3 & $\ldots$ & $\ldots$ \\
\hline IL-4 & 1 & $1.02(1.00-1.03)$ & 0.054 \\
\hline & 2 & $\ldots$ & $\ldots$ \\
\hline & 3 & $\ldots$ & $\ldots$ \\
\hline IL-6 & 1 & $1.63(1.03-2.44)$ & 0.017 \\
\hline & 2 & $1.96(1.14-6.60)$ & 0.020 \\
\hline & 3 & $1.83(1.08-6.36)$ & 0.043 \\
\hline IL-10 & 1 & $1.15(1.03-1.28)$ & 0.011 \\
\hline & 2 & $1.17(1.04-1.31)$ & 0.009 \\
\hline & 3 & $1.15(1.02-1.30)$ & 0.019 \\
\hline TNF & 1 & $1.21(0.80-1.83)$ & 0.359 \\
\hline & 2 & $\ldots$ & $\ldots$ \\
\hline & 3 & $\ldots$ & $\ldots$ \\
\hline INF & 1 & $0.86(0.52-1.41)$ & 0.548 \\
\hline & 2 & $\ldots$ & $\ldots$ \\
\hline & 3 & $\ldots$ & $\ldots$ \\
\hline
\end{tabular}

END, early neurological deterioration; IL, interleukin; TNF, tumor necrosis factor; INF, interferon; OR, odds ratio.

${ }^{a}$ Model 1: unadjusted; model 2: adjusted for age and sex; model 3 adjusted for model 2 with atrial fibrillation.

\section{Figures}




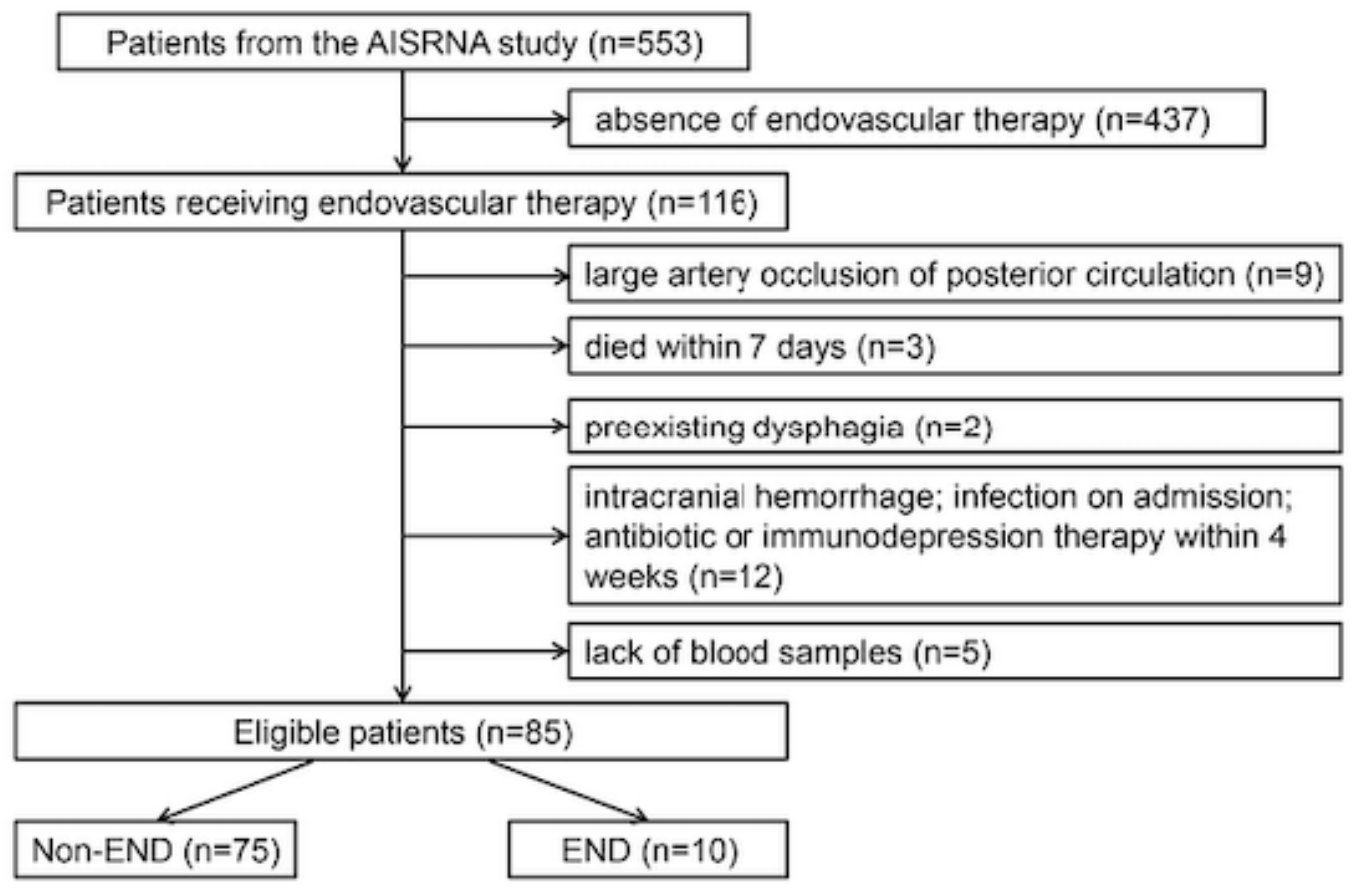

\section{Figure 1}

Flowchart of the study patients to illustrate study screening, recruitment, and follow-up. END, early neurological deterioration. 

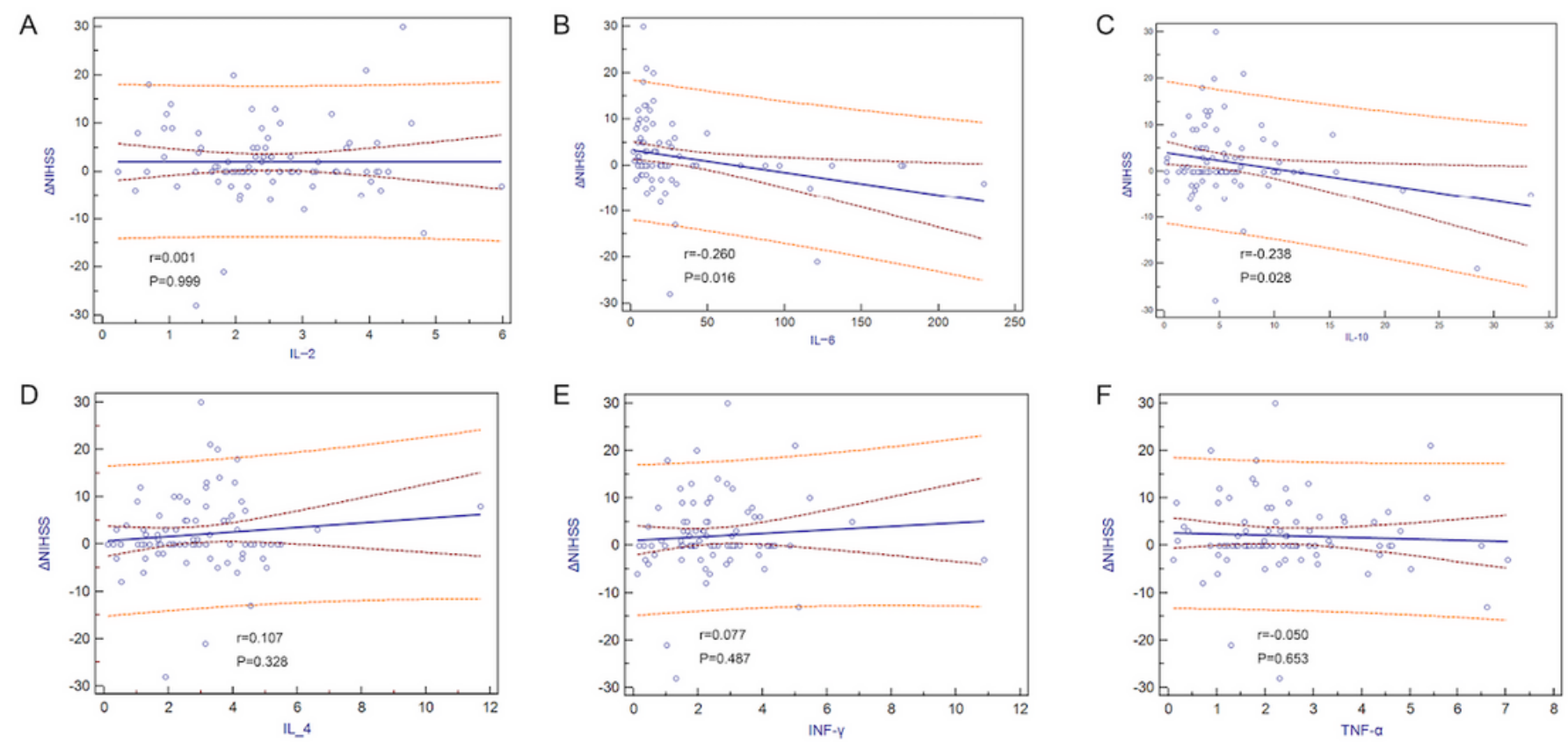

Figure 2

A correlation analysis to investigate the correlation between IL-2 (A), IL-6 (B), IL-10 (C), IL-4 (D), INF-y (E), and TNF-a (F) and delta NIHSS 0-24h. 


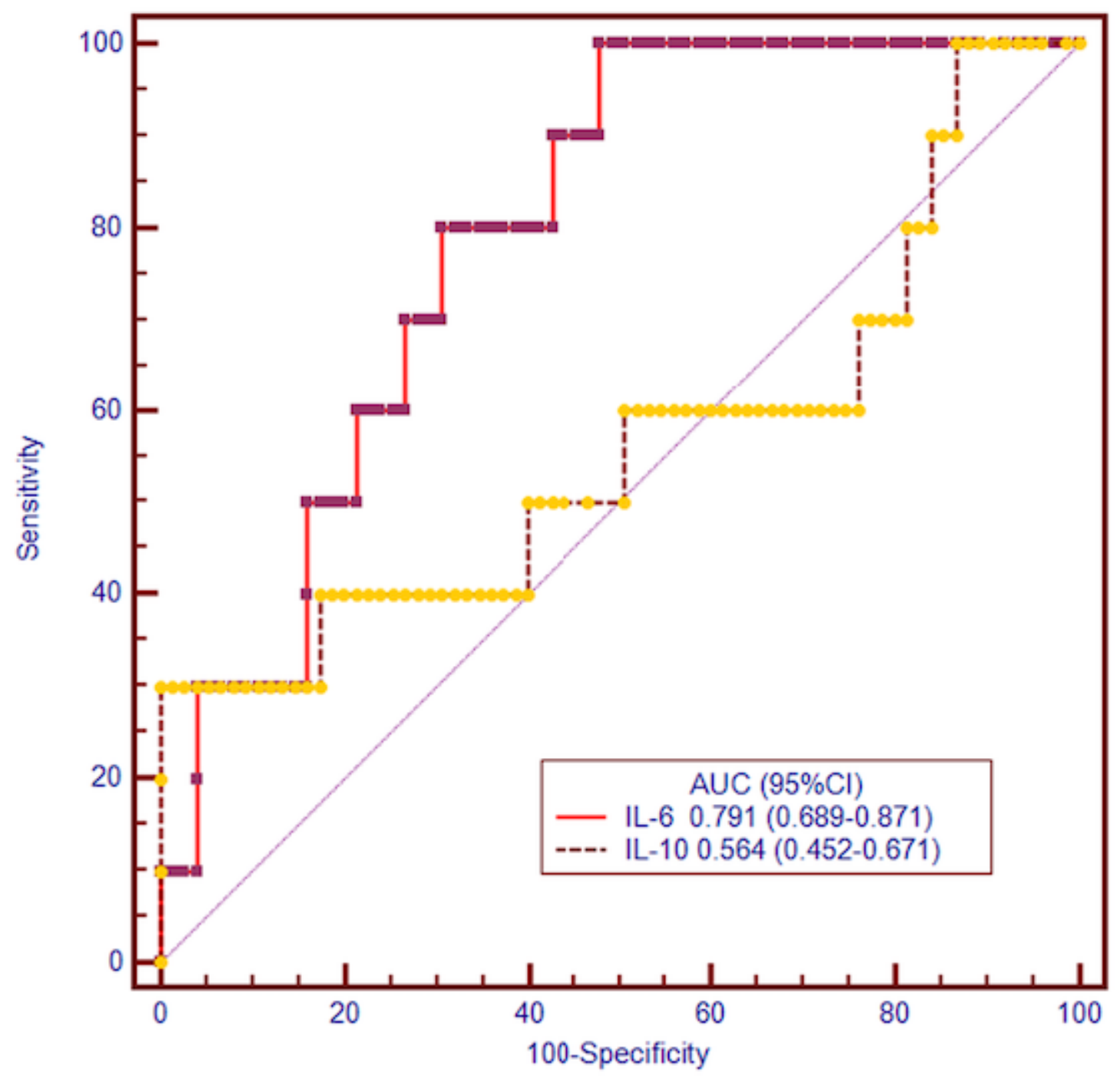

Figure 3

Discriminatory capacities of IL- 6 and IL-10 for END after EVT. The area under curves of IL- 6 and IL-10 for predicting END were 0.791 (0.689-0.871), and 0.564 (0.452-0.671), respectively $(P<0.001)$. END, early neurological deterioration; EVT, endovascular therapy. 
A

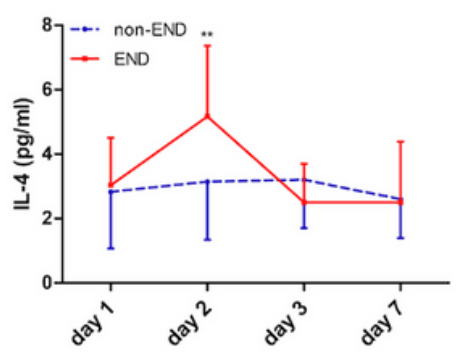

D

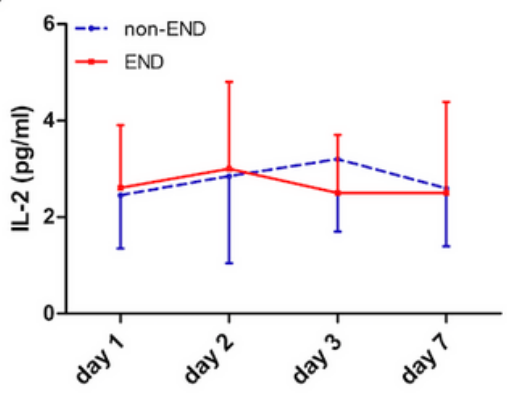

B

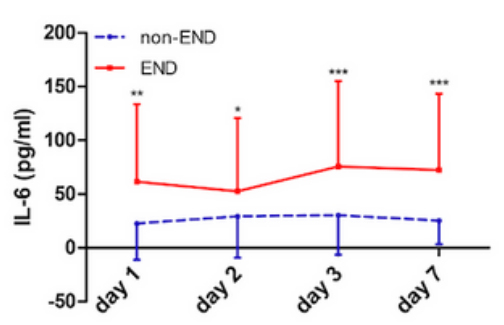

$\mathrm{E}$

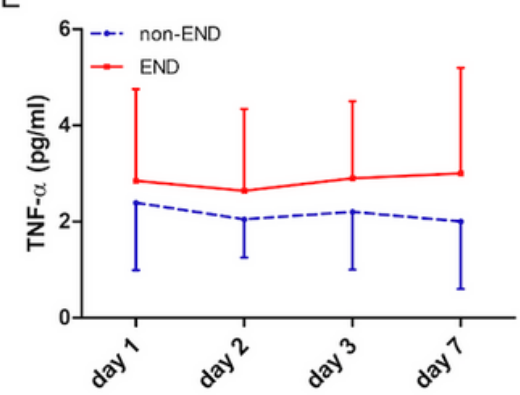

C

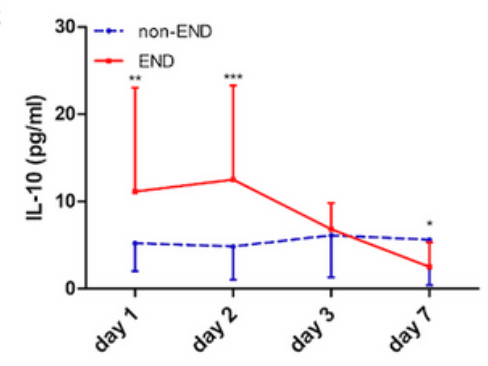

$\mathrm{F}$

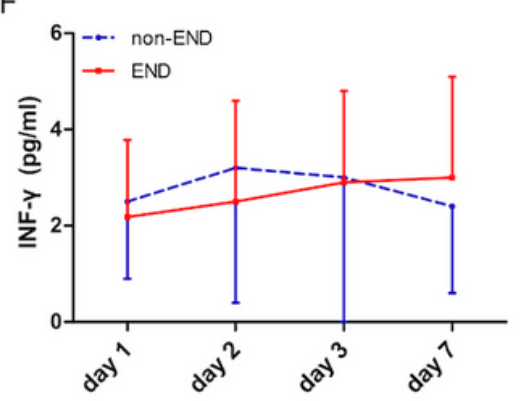

\section{Figure 4}

Time course of inflammatory factors after stroke onset (days 1 to 7 ). Significant changes over time were observed in the expression levels of IL-4, IL-6, and IL-10 in patients undergoing END compared with nonEND $(P<0.05)$. IL-6 levels were obviously increased from days 1 to 7 after EVT (B). IL-4 (A) and IL-10 (C) peak levels were at 2 days, and then rapidly decreased at 3 days. END, early neurological deterioration. ${ }^{*} P<0.05$, ${ }^{\star *} P<0.01$, ${ }^{* \star *} P<0.001$. 